\title{
The Role of Atorvastatin in Suppressing Fibroid Related Symptom and Fibroid Size in Perimenopausal Women
}

\author{
Ahmed Elsayed Abdel Aleem Seada, Yasser Abdel Dayem Elmorsy, \\ Emad Ahmed Fayala, Ehsan Mohamed Raghib Refaie
}

Department of Obstetrics and Gynecology, Faculty of Medicine, Mansoura University, Mansoura, Egypt

*Corresponding author: Ahmed Elsayed Abdel Aleem Seada, Mobile: (+20)01025020643,

E-Mail: ahmedseada1201011@gmail.com

\begin{abstract}
Background: Uterine fibroids are the most common benign gynecologic tumors, with a lifetime incidence of approximately $70 \%$. They are associated with multiple symptoms, including heavy uterine bleeding and pelvic pain. Currently used treatments include contraceptive steroids, Gonadotropin-releasing hormone agonist (GnRHa), progesterone modulators, uterine artery embolization (UAE), magnetic resonance imaging-guided focused ultrasound surgery (MRgFUS), and radiofrequency ablation (RFA).
\end{abstract}

Objective: To evaluate the effect of atorvastatin on decreasing fibroid related symptoms and fibroid size, endometrial thickness and cholesterol level in perimenopausal women.

Patients and methods: Prospective randomized controlled trial that was conducted over a period of 12 months during 2019 and 2021 on 105 patients with symptomatic uterine fibroid in perimenopausal age, admitted to emergency unit and gynecology outpatient clinic at Mansoura University Hospital and after obtaining acceptance from institutional research board faculty of medicine then patients were prospectively recruited and randomized by computer program. Results: All groups at presentation demonstrated insignificant differences concerning demographic characteristics, gravidity and symptoms of uterine fibroid. Uterine fibroid demonstrated significant reduction in size as well as significant changes following the atorvastatin treatment after 6 months in a dose dependent manner, while endometrial thickness demonstrated insignificant differences as measured by transvaginal ultrasound (TVS). Cholesterol level demonstrated highly statistically significant decrease following atorvastatin treatment in a dose dependent manner.

Conclusion: The current study demonstrated that atorvastatin has promising effects in cases with uterine fibroids as it was associated with a reduction of fibroid size with reduction of fibroid related symptom (menorrhagia, dysmenorrhea). Keywords: Atorvastatin, Perimenopausal Women, Uterine fibroids

\section{INTRODUCTION}

Uterine fibroids are the most common benign tumor in women, with a lifetime incidence of approximately $70 \%$. It is associated with variety of problems including menorrhagia, pelvic pain, pelvic pressure, as well as infertility and pregnancy complication ${ }^{(1,2)}$.

Current therapeutic options mainly involve surgical interventions to remove or destroy uterine fibroids. However, these procedures have significant morbidity and mortality, which are the major limitations of surgical intervention. To avoid the risks of surgical intervention many novel therapies are currently under investigation. At present, medical interventions used to manage uterine fibroids include mifepristone, ulipristal acetate, and GnRH-agonists. Nevertheless, uterine fibroids will regrow fast after the cessation of mifepristone, and the duration of treatment with GnRH-agonists is limited by the induction of hypoestrogenic symptoms ${ }^{(3-5)}$.

Medical therapeutic options are quite limited for uterine fibroids treatment. Therefore, it is of great clinical value to develop new treatment strategy for uterine fibroids. Statins are a drug family primarily used for hyperlipidemia. By inhibiting 3-hydroxy-3methylglutaryl coenzyme A (HMG-CoA) reductase, statins prevent the conversion of $\mathrm{HMG}-\mathrm{CoA}$ to mevalonate, and thus lead to dramatic reductions in both cholesterol and its isoprenoid precursors; farnesyl pyrophosphate (FPP) and geranylgeranyl pyrophosphate (GGPP) ${ }^{(3)}$.

Statins can inhibit the production of 2 endogenous cholesterol and block protein prenylation, and thus statins use may therefore influence cell proliferation and migration. An increasing evidence suggests that statins use related to several potential anticancer properties and a reduced risk of cancer recurrence, such as hepatocellular cancer, multiple myeloma, breast cancer and ovarian cancer ${ }^{(2,6)}$.

It was reported that the use of statins was related to a lower risk of uterine fibroids and fibroidcaused symptoms. Simvastatin and atorvastatin inhibit progression of certain benign steroid dependent gynecological disorders such as endometriosis. Statins also inhibit proliferation and induce calcium dependent programmed cell death in human uterine fibroids cells in vitro and in vivo ${ }^{(7)}$.

Atorvastatin is the most widely used antihyperlipidemia, and its effect on fibroid growth remains unknown. Therefore, in the present study, we proceeded to investigate therapeutic effects of atorvastatin on uterine fibroids.

The aim of the current study was to evaluate the effect of atorvastatin on decreasing fibroid related symptoms and fibroid size, endometrial thickness and cholesterol level in perimenopausal women. 


\section{PATIENTS AND METHODS}

Prospective randomized controlled trial was conducted over a period of 12 months between 2019 and 2021 on 105 patients admitted to emergency unit and gynecology outpatient clinic at Mansoura University Hospital.

Inclusion criteria: Age range 45-51 years old, diagnosed as symptomatized uterine fibroid, and completing her family.

Exclusion criteria: Hypersensitivity to atorvastatin, taking any medication that affects uterine fibroid within last 6 months, using any hormonal contraception, and association with any medical disorder, e.g. liver disease, renal disease, and thyroid disorder or muscle weakness.

\section{Sample Size calculation;}

The calculated sample size of the study was 29 participants for each group at 5\% level of significance and $90 \%$ power. It was increased to 35 participants for each group to compensate for protocol failures with incomplete data and to increase the study power.

\section{Entire cases were subjected to the following:}

I) Full History taking: Personal history, menstrual history, obstetric history, past history, family history.

II) Presenting symptoms: which included menorrhagia and dysmenorrhea.

III) Physical examination: which included general and abdominal examinations.

IV) The method of initial diagnosis :

- Transabdominal US (TAS): determined type and size of uterine fibroid.

- TVS: determined type and size of uterine fibroid and endometrial thickness.

- Size of uterine fibroid both before and after atorvastatin treatment.

- The length (d1), width (d2) and depth (d3) of each tumor were measured, and the volumes were calculated by the following formula: volume $(\mathrm{cm} 3)=0.52 \times \mathrm{d} 1 \times \mathrm{d} 2 \times \mathrm{d} 35$.

- Laboratory investigation: $\mathrm{CBC}$, Cholesterol level, Liver function test, Kidney function test, Thyroid profile.

\section{Thereafter:}

A total of 105 patients were prospectively recruited and randomized by computer program (double blinded method) to 3 groups by receiving "concealed envelope":

- Group A: receiving atorvastatin $20 \mathrm{mg}$ for 6 month.

- Group B: receiving atorvastatin $40 \mathrm{mg}$ for 6 month.

- Control group: receiving placebo for 6 month.

All patients received Daflon 1x2 for 1 week with every menses.

Follow up:

Patients were re-examined after 3 months and 6 months at the end of study regarding to:
1) Interview and clinical evaluation of symptoms.

2) US : TAS and TVS for follow up of fibroid size and endometrial thickness:

- Initial evaluation: Before administration of drug.

- Following up evaluation: 3 months after administration of drug.

- Last evaluation: after completing administration of drug.

3) Follow up and evaluation symptoms regarding pain and bleeding before and after administration of drug.

4) Cholesterol level before and after administration of drug. The protocols were compared regarding the overall response rate i.e., fibroid size and fibroid related symptom.

\section{Ethical Consideration:}

AII women were counseled by an experienced gynecologist and they provided informed written and valid consent before entering the study. The research approval of the study was obtained from Institutional Research Board (IRB) of Faculty of Medicine at Mansoura University before starting the study. This work has been carried out in accordance with The Code of Ethics of the World Medical Association (Declaration of Helsinki) for studies involving humans.

The researcher clarified the objective and aim of the study to the subjects included in the study. The researcher assured maintaining anonymity and confidentiality of subjects' data. Subjects were informed that they are allowed to choose to participate or not in the study and that they had the right to withdraw from the study at any time without giving any reasons. Ethics, values, culture and beliefs of subjects were respected.

\section{Statistical analysis}

Data were analyzed using the Statistical Package for the Social Sciences (SPSS) program for Windows (Standard version 21). The normality of data was first tested with one-sample Kolmogorov-Smirnov test. Qualitative data were described using number and percent. Association between categorical variables was tested using Chi-square test. Continuous variables were presented as mean \pm SD (standard deviation) for normally distributed data and median (min-max) for non-normal data. Quantitative data were compared by ANOVA test when normally distributed or by KruskalWallis test when abnormally distributed, and when the difference was significant; post hoc test was used for multiple comparison. The results was considered significant when $\mathrm{p} \leq 0.05$.

\section{RESULTS}

Table (1) shows that there was no statistically significant difference in the demographic characters among the three studied groups as regard, age, BMI, occupation, residency and family history of uterine fibroid. 
Table (1): Demographic characteristics of the studied groups

\begin{tabular}{|c|c|c|c|c|c|}
\hline the & 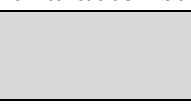 & $\begin{array}{c}\text { Group A } \\
(\mathbf{n}=\mathbf{3 5})\end{array}$ & $\begin{array}{c}\text { Group B } \\
(\mathbf{n}=35)\end{array}$ & $\begin{array}{c}\text { Control group } \\
(\mathrm{n}=35)\end{array}$ & $\mathbf{P}$ \\
\hline \multicolumn{2}{|c|}{ Age (years) } & $48.89 \pm 1.728$ & $48.37 \pm 1.536$ & $48.49 \pm 1.560$ & $\begin{array}{l}\mathrm{F}=0.984 \\
\mathrm{p}=0.377\end{array}$ \\
\hline \multicolumn{2}{|c|}{ BMI (kg/m2) } & $30.29 \pm 2.866$ & $30.50 \pm 3.037$ & $29.82 \pm 2.103$ & $\begin{array}{l}\mathrm{F}=0.587 \\
\mathrm{P}=0.558\end{array}$ \\
\hline \multirow{2}{*}{ Occupation } & Housewife & $23(65.7 \%)$ & $18(51.4 \%)$ & $21(60.0 \%)$ & \multirow{2}{*}{$\begin{array}{l}\chi^{2}=1.497 \\
p=0.473\end{array}$} \\
\hline & Worker & $12(34.3 \%)$ & $17(48.6 \%)$ & $14(40.0 \%)$ & \\
\hline \multirow{2}{*}{ Residency } & Urban & $14(40.0 \%)$ & $19(54.3 \%)$ & $22(62.9 \%)$ & \multirow{2}{*}{$\begin{array}{l}\chi^{2}=3.742 \\
p=0.154\end{array}$} \\
\hline & Rural & $21(60.0 \%)$ & $16(45.7 \%)$ & $13(37.1 \%)$ & \\
\hline \multicolumn{2}{|c|}{$\begin{array}{l}\text { Family history of uterine } \\
\text { fibroid }\end{array}$} & $9(25.7 \%)$ & $8(22.9 \%)$ & $7(20.0 \%)$ & $\begin{array}{l}\chi^{2}=0.324 \\
p=0.850\end{array}$ \\
\hline
\end{tabular}

Table (2) shows that there was no statistically significant difference in parity and gravidity among the three studied groups.

Table (2): Parity and gravidity in the studied groups

\begin{tabular}{|c|c|c|c|c|}
\hline & $\begin{array}{c}\text { Group A } \\
(\mathbf{n}=\mathbf{3 5})\end{array}$ & $\begin{array}{c}\text { Group B } \\
(\mathbf{n}=\mathbf{3 5})\end{array}$ & $\begin{array}{c}\text { Control group } \\
(\mathrm{n}=35)\end{array}$ & $\mathbf{P}$ \\
\hline $\begin{array}{c}\text { Parity } \\
\text { Median (Min-max) }\end{array}$ & $2(0-4)$ & $2(0-3)$ & $2(0-3)$ & $\begin{array}{c}\mathrm{KW}=1.71 \\
\mathrm{P}=0.425\end{array}$ \\
\hline $\begin{array}{c}\text { Gravidity } \\
\text { Median (Min-max) }\end{array}$ & $3(1-5)$ & $3(1-5)$ & $3(1-5)$ & $\begin{array}{c}\mathrm{KW}=1.01 \\
\mathrm{P}=0.603\end{array}$ \\
\hline
\end{tabular}

Table (3) shows that there was improvement of menorrhagia in treated group (A and B) compared to control group with statistically significant difference after 6 months of treatment, and there was slight improvement with higher dose of atorvastatin (group B) without reaching the statistical significant difference.

There was slight improvement of dysmenorrhea in treated group compared to control group without reaching the statistical difference after 6 months of treatment and there was slight improvement with higher dose of atorvastatin (group B) without reaching the statistical significant difference.

Table (3): Symptoms of uterine fibroid at presentation and after 6 month in the studied groups

\begin{tabular}{|c|c|c|c|c|c|c|c|}
\hline 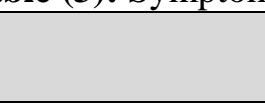 & $\begin{array}{c}\text { Group A } \\
(\mathbf{n}=\mathbf{3 5})\end{array}$ & $\begin{array}{c}\text { Group B } \\
(\mathbf{n}=35)\end{array}$ & $\begin{array}{c}\text { Control group } \\
(n=35)\end{array}$ & $\mathbf{p}$ & P1 & P2 & P3 \\
\hline $\begin{array}{c}\text { Menorrhagia at } \\
\text { presentation }\end{array}$ & $\begin{array}{c}23 \\
(65.7 \%) \\
\end{array}$ & $\begin{array}{c}27 \\
(77.1 \%) \\
\end{array}$ & $25(71.4 \%)$ & $\begin{array}{l}\chi^{2}=1.12 \\
P=0.57\end{array}$ & - & - & - \\
\hline $\begin{array}{l}\text { Menorrhagia } \\
\text { after } 6 \text { month }\end{array}$ & $9(25.7 \%)$ & $8(22.8 \%)$ & $23(65.7 \%)$ & $\begin{array}{l}\chi^{2}=17.04 \\
\mathbf{P}<\mathbf{0 . 0 0 1}\end{array}$ & $\begin{array}{l}\chi^{2}=0.08 \\
P=0.78\end{array}$ & $\begin{array}{l}\chi^{2}=11.28 \\
\mathbf{P}=\mathbf{0 . 0 0 1}\end{array}$ & $\begin{array}{l}\chi^{2}=13.03 \\
\mathbf{P}<\mathbf{0 . 0 0 1}\end{array}$ \\
\hline Improved cases & $\begin{array}{c}14 \\
(60.9 \%)\end{array}$ & $\begin{array}{c}19 \\
(70.4 \%)\end{array}$ & $2(8 \%)$ & $\begin{array}{l}\chi^{2}=22.97 \\
\mathbf{P}<\mathbf{0 . 0 0 1}\end{array}$ & $\begin{array}{l}\chi^{2}=0.50 \\
P=0.48\end{array}$ & $\begin{array}{l}\chi^{2}=15.07 \\
\mathbf{P}<\mathbf{0 . 0 0 1}\end{array}$ & $\begin{array}{l}\chi^{2}=20.97 \\
\mathbf{P}<\mathbf{0 . 0 0 1}\end{array}$ \\
\hline $\begin{array}{l}\text { dysmenorrhea } \\
\text { at presentation }\end{array}$ & $\begin{array}{c}16 \\
(45.7 \%) \\
\end{array}$ & $\begin{array}{c}12 \\
(34.3 \%) \\
\end{array}$ & $10(28.6 \%)$ & $\begin{array}{l}\chi^{2}=2.31 \\
\mathrm{P}=0.315\end{array}$ & - & - & - \\
\hline $\begin{array}{l}\text { dysmenorrhea } \\
\text { after } 6 \text { month }\end{array}$ & $8(22.8 \%)$ & $5(14.3 \%)$ & $9(25.7 \%)$ & $\begin{array}{l}\chi^{2}=1.49 \\
P=0.474\end{array}$ & - & - & - \\
\hline Improved cases & $8(50 \%)$ & $7(58.3 \%)$ & $1(10 \%)$ & $\begin{array}{l}\chi^{2}=5.93 \\
\mathbf{P}=\mathbf{0 . 0 5}\end{array}$ & $\begin{array}{l}\chi^{2}=0.19 \\
P=0.66\end{array}$ & $\begin{array}{l}\chi^{2}=4.35 \\
\mathbf{P}=\mathbf{0 . 0 3 7}\end{array}$ & $\begin{array}{l}\chi^{2}=5.51 \\
\mathbf{P}=\mathbf{0 . 0 1 9}\end{array}$ \\
\hline
\end{tabular}

Data are expressed as frequency and percentage. P1: Group A vs group B. P2: Group A vs control group. P3:

Group B vs control group.

Table (4) shows that as regard uterine fibroid size there was reduction in size more in treated group (A and B) compared with control group with statistically significant difference after 6 months. There was statistically significant difference between group B and group A in reduction in size with increase dose of atorvastatin. As regard endometrial thickness there was no statistically significant difference between three groups after 6 months of treatment. 
Table (4): Uterine fibroid size follow-up and endometrial thickness in the current study as measured by TVS

\begin{tabular}{|c|c|c|c|c|c|c|c|}
\hline TVS & $\begin{array}{c}\text { Group A } \\
(\mathbf{n}=\mathbf{3 5})\end{array}$ & $\begin{array}{c}\text { Group B } \\
(\mathbf{n}=35)\end{array}$ & $\begin{array}{l}\text { Control } \\
\text { group } \\
(n=35)\end{array}$ & $\mathbf{P}$ & P1 & P2 & P3 \\
\hline $\begin{array}{c}\text { Size at } \\
\text { presentation }\end{array}$ & $4.66 \pm 1.11$ & $4.65 \pm 1.03$ & $4.54 \pm 0.78$ & $\begin{array}{l}F=0.195 \\
P=0.823\end{array}$ & - & - & - \\
\hline Size at 3 months & $4.15 \pm 1.11$ & $4.15 \pm 1.03$ & $4.06 \pm 1.05$ & $\begin{array}{c}\mathrm{F}=0.267 \\
\mathrm{P}=0.78\end{array}$ & - & - & - \\
\hline Size at 6 months & $4.01 \pm 0.64$ & $3.49 \pm 0.39$ & $4.58 \pm 0.86$ & $\begin{array}{l}F=26.73 \\
P<0.001\end{array}$ & $\begin{array}{c}t=4.13 \\
P<0.001\end{array}$ & $\begin{array}{c}t=3.46 \\
P=0.001\end{array}$ & $\begin{array}{c}t=7.19 \\
P<0.001\end{array}$ \\
\hline $\begin{array}{l}\text { Endometrial } \\
\text { thickness }\end{array}$ & $6.77 \pm 2.48$ & $6.34 \pm 2.81$ & $6.68 \pm 2.64$ & $\begin{array}{l}\mathrm{F}=0.256 \\
\mathrm{P}=0.775\end{array}$ & - & - & - \\
\hline
\end{tabular}

Table (5) shows that as regard cholesterol level there was statistically significant difference between the three groups after 6 months of treatment. There was statistically significant difference between (group A and B), (group A and control group) and (group B and control group) after 6 months.

Table (5) Cholesterol levels in the current study

\begin{tabular}{|c|c|c|c|c|c|c|c|}
\hline $\begin{array}{c}\text { Cholesterol } \\
\text { level }\end{array}$ & $\begin{array}{c}\text { Group A } \\
(\mathbf{n}=35)\end{array}$ & $\begin{array}{c}\text { Group B } \\
(\mathbf{n}=35)\end{array}$ & $\begin{array}{c}\text { Control group } \\
(n=35)\end{array}$ & $\mathbf{P}$ & P1 & $\mathbf{P 2}$ & $\mathbf{P 3}$ \\
\hline Presentation & $164.77 \pm 19.182$ & $172.37 \pm 13.599$ & $166.37 \pm 9.828$ & $\begin{array}{l}\mathrm{F}=2.59 \\
\mathrm{P}=0.08\end{array}$ & - & - & - \\
\hline At 6 months & $133.29 \pm 21.296$ & $111.91 \pm 23.788$ & $164.71 \pm 19.038$ & $\begin{array}{c}F=53.6 \\
P<0.001\end{array}$ & $\begin{array}{c}t=3.96 \\
P<0.001 \\
\end{array}$ & $\begin{array}{c}\mathrm{t}=6.51 \\
\mathrm{P}<0.001\end{array}$ & $\begin{array}{l}\mathrm{t}=\mathbf{1 0 . 2 5} \\
\mathrm{P}<\mathbf{0 . 0 0 1} \\
\end{array}$ \\
\hline
\end{tabular}

\section{DISCUSSION}

The present study included 105 patient randomized into 3 groups: (group A: receiving atorvastatin $20 \mathrm{mg}$ for 6 month), (group B: receiving atorvastatin $40 \mathrm{mg}$ for 6 month) and (control group receiving placebo for 6 month).

The present study found that there was no statistically significant difference in the demographic characters among the three studied groups as regard, age, BMI, occupation, residency and family history of uterine fibroid. Also, the present study found that there was no statistically significant difference in parity and gravidity among the three studied groups. Additionally the study found that there was improvement of menorrhagia in treated group (A and $\mathrm{B}$ ) compared to control group with statistically significant difference after 6 months of treatment and there was slight improvement with higher dose of atorvastatin (group B) without reaching the statistical difference.

As regard dysmenorrhea there was slight improvement in treated group compared to control group without reaching the statistical significant difference after 6 months of treatment and there was slight improvement with higher dose of atorvastatin (group B) without reaching the statistical significant difference. In agreement with these findings, Borahay et al. ${ }^{\left({ }^{(8)}\right.}$ who found that statin use was associated with significant improvement of menorrhagia, anemia, and pelvic pain. They also found that the fibroid-associated symptoms of menorrhagia, anemia, and pelvic pain were significantly lower among statin users compared to nonusers. Also Shen et al. ${ }^{(9)}$ found that beneficial association of statins on fibroid-related symptoms is promising.

Moreover, the current study evaluated the uterine fibroid size by TVS. At the time of presentation and also after 3 months of treatment, there was no statistically significant difference between three groups. However, there was decrease of uterine fibroid size more in treated group (A and B) compared with control group with statistically significant difference after 6 months and the size decreased by $14.6 \%$ in group A and $23.8 \%$ in group B and there was statistically significant difference between group B and group $\mathrm{A}$ in reduction of size with increased dose of atorvastatin. Additionally, there was no statistically significant difference between three groups as regard endometrial thickness. In agreement with the above results, Shen et al. (9) found that no significant differences were noted in the initial tumor size between the study group and control group $(\mathrm{P}>0.05)$.

Fibroid volume was determined after treated with or without atorvastatin for 1 and 2 years. While the fibroid volume of control group was increased gradually, the study group experienced stable volume. Totally, after 1 year follow-up, the fibroid volume of 
26 cases $(49.1 \%)$ was decreased in study group, while in the control group there were only 12 cases $(17.9 \%)$ presenting reduction volume. After 2 years follow-up, the fibroid volume was decreased in 28 cases $(52.8 \%)$ of study group and 10 cases $(14.9 \%)$ of control group. Furthermore, volume change of uterine fibroids was determined after follow-up for 1 or 2 years. Volume change of uterine fibroids was much less in study group as compared to control group. Collectively, atorvastatin used for 1 or 2 years significantly suppressed growth of human uterine fibroids.

Malik et al. ${ }^{(10)}$ performed a study to observe the anti-fibroid effects of therapeutic concentrations of simvastatin in the development and growth of uterine leiomyomas. They concluded that inhibition of cellular proliferation with treatment can be caused either through cytostatic effect, cellular necrosis, or induction of apoptotic pathway. They observed that induction of the activation of caspase- 3 apoptotic pathway occur at lower concentration, which became significant at all higher concentrations of simvastatin.

The current study has showed that there was no statistically significant difference between the three groups as regard cholesterol blood level at time of presentation. However, there was statistically significant difference between the three groups as regard cholesterol blood level after 6 months from receiving the drug, where group $B$ showed the lowest cholesterol level at 6 months after treatment followed by group A then control group. Similarly, Demierre et al. (11) illustrated that atorvastatin reduces serum cholesterol levels mainly by competitively inhibiting HMG-CoA reductase, the rate-limiting enzyme in the mevalonate synthesis pathway.

Statins are a drug family basically used for hyperlipidemia. They inhibit 3-hydroxyl-3methyl glutaryl coenzyme A (HMGCoA) reductase and hence prevent the conversion of HMG-CoA to mevalonate and hence there is dramatic decreases in both cholesterol and its isoprenoid precursors; farnesyl pyrophosphate (FPP) and geranyl geranyl pyrophosphate (GGPP) ${ }^{(\mathbf{1 2})}$.

There were no detected cases with side effects of atorvastatin e.g., statin-associated myalgias, hepatotoxicity, nephrotoxicity or hyperglycemia.

After the use of atorvastatin for 6 months, there was a remarkable improvement in the size of fibroids and associated symptoms, and we need more research on more cases and longer duration to demonstrate the effectiveness of the drug in the treatment of fibroids.

\section{CONCLUSION}

The current study demonstrated that, atorvastatin has promising effects in cases with uterine fibroids as it was associated with a reduction of fibroid size with reduction of fibroid related symptom (menorrhagia, dysmenorrhea). In addition reduction of size occur in a dose dependent manner (reaching the statistical significance with increase dose) but decrease menorrhagia and dysmenorrhea occur in a dose dependent manner (not reaching the statistical significance with increase dose).

\section{REFERENCES}

1. Bulun S (2013): Uterine fibroids. New England Journal of Medicine, 369(14): 1344-1355.

2. Styer A, Rueda B (2016): The epidemiology and genetics of uterine leiomyoma. Best Practice \& Research Clinical Obstetrics \& Gynaecology, 34: 3-12.

3. Armitage $\mathbf{J}$ (2007): The safety of statins in clinical practice. The Lancet, 370(9601): 1781-1790.

4. Shen Y, Xu Q, Ren M et al. (2013): Measurement of phenolic environmental estrogens in women with uterine leiomyoma. PloS One, 8(11): 79838-43.

5. Shen Q, Shu L, Luo H et al. (2017): The use of mifepristone in abortion associated with an increased risk of uterine leiomyomas. Medicine, 96 (17): 6680-6686.

6. Nielsen S, Nordestgaard B, Bojesen S (2012): Statin use and reduced cancer-related mortality. New England Journal of Medicine, 367(19): 1792-1802.

7. Borahay M, Fang X, Baillargeon J et al. (2016): Statin use and uterine fibroid risk in hyperlipidemia patients: a nested case-control study. American Journal of Obstetrics and Gynecology, 215(6): 750. e751-750. e758.

8. Borahay M, Kilic G, Yallampalli C et al. (2014): Simvastatin potently induces calcium-dependent apoptosis of human leiomyoma cells. Journal of Biological Chemistry, 289(51): 35075-35086.

9. Shen Z, Li S, Sheng B et al. (2018): The role of atorvastatin in suppressing tumor growth of uterine fibroids. Journal of Translational Medicine, 16(1): 1-13.

10. Malik M, Britten J, Borahay $M$ et al. (2018): Simvastatin, at clinically relevant concentrations, affects human uterine leiomyoma growth and extracellular matrix production. Fertility and sterility, 110(7): 13981407.

11.Demierre M, Higgins P, Gruber et al. (2005): Statins and cancer prevention. Nature Reviews Cancer, 5(12): 930-942.

12. Kaur K, Allahbadia G, Singh M (2019): Use of $20 \mathrm{mg}$ statins (atorvastatin/simvastatin) as a novel new option of medically treating fibroids-overcoming the drawback of selective progesterone receptor modulators of interruption before long term use. Int J Pregn \& Chi Birth, 5(3): 116-117. 